\title{
ON DETECTING SOFT LAYERS IN THE MANTLE WITH RAYLEIGH WAVES
}

\author{
By D. G. Harkrider, A. L. Hales, and F. Press
}

\begin{abstract}
The feasibility of an experiment to detect soft or molten zones in the mantle using Rayleigh wave phase velocities is examined. It is found that when the anomalous zone has large lateral extent the phase velocity curves are so affected as to make it easily detectable. A discussion of dispersion curves and displacement with depth is presented. A pseudo-flexural mode of oscillation is found to be possible for the layers above a molten zone.
\end{abstract}

\section{INTRODUCTION}

The power of surface wave methods of exploring the earth's interior was demonstrated recently with the verification of the Gutenberg low velocity zone of the upper mantle as a world-wide phenomenon (Dorman, Ewing, and Oliver, 1960).

This paper investigates the sensitivity of Rayleigh wave dispersion curves to the presence of soft and liquid layers of various thicknesses and depths in the upper mantle. If Rayleigh wave phase velocities are found to respond to a measurable degree to the presence of such anomalous zones in the mantle, then an experiment for detecting such zones becomes feasible. The importance of an experimental method for locating magma chambers, or partially molten zones in the mantle, cannot be overstated. The nature of volcanism remains a fundamental problem in geology and progress towards its solution will be impeded until the source of the primary magmas is found and described. Hales (1960) has investigated other methods for detecting weak layers in the mantle.

The procedure we will follow is to compute dispersion curves and displacements at depth for various models of the crust and mantle. The computation has been programmed earlier for the IBM 7090 computer (Press, Harkrider, and Seafeldt, 1962; Harkrider and Anderson, 1962). Since the computation assumes that the earth may be represented by plane parallel layers of infinite horizontal extent, the results apply to the detection of soft zones having lateral extent large compared to a wavelength of the phase velocity used. The calculations have not allowed for the spherical curvature of the earth since we were primarily interested in exploring the effects of soft zones rather than advancing a particular model.

\section{Dispersion Curves}

The crust-mantle models used in this study are presented in tables 1 and 2 . Phase velocity curves for the various models are compared with the standard "Gutenberg" continental model in figures 1-3. "Soft" zones were represented by layers of lower compressional and shear velocity than the standard model. Molten zones were represented by layers with vanishing shear velocity. Density was held to the constant value 3.24 for all of the soft layers. Thickness and depth of the layers were also varied. The parameters shown in figures 1-3 are the shear velocity (or compressional velocity in the case of a liquid layer), the depth to the layer mid point and the layer thickness. In the following discussion the term "deviation from 
the normal case" will mean the difference in phase velocity between the case considered and the Gutenberg case, 21, for a given period.

For all cases, the effect of inserting a low velocity layer in the velocity depth distribution was to lower the phase velocity curves. For the cases calculated, the relative effects caused by differences of velocity, thickness, and depth are as follows:

1. Increasing the layer thickness (without increasing the overall section thickness) while keeping the depth and velocity constant caused a greater deviation from the

TABLE 1

Gutenberg Moder Case 21

$d$-Layer thicknesses in kilometers.

$V_{p}-$ Compressional velocities in $\mathrm{km} / \mathrm{sec}$.

$V_{s}$-Shear velocities in $\mathrm{km} / \mathrm{sec}$.

$\rho$-Densities in $\mathrm{g} / \mathrm{cm}^{3}$.

$T M P$-Total depth to mid point of layer in kilometers.

\begin{tabular}{r|c|c|c|c}
\hline$d$ & $V_{p}$ & $V_{s}$ & $\rho$ & $T M P$ \\
\hline 22 & 6.03 & 3.53 & 2.78 & 11.0 \\
15 & 6.70 & 3.80 & 3.00 & 29.5 \\
13 & 7.96 & 4.60 & 3.37 & 43.5 \\
25 & 7.85 & 4.50 & 3.39 & 62.5 \\
50 & 7.85 & 4.41 & 3.42 & 100.0 \\
75 & 8.00 & 4.41 & 3.45 & 162.5 \\
50 & 8.20 & 4.50 & 3.47 & 225.0 \\
100 & 8.40 & 4.60 & 3.50 & 300.0 \\
100 & 9.00 & 4.95 & 3.63 & 400.0 \\
100 & 9.63 & 5.31 & 3.89 & 500.0 \\
100 & 10.17 & 5.63 & 4.13 & 600.0 \\
100 & 10.585 & 5.915 & 4.33 & 700.0 \\
100 & 10.955 & 6.14 & 4.49 & 800.0 \\
100 & 11.275 & 6.285 & 4.60 & 900.0 \\
150 & 11.46 & 6.384 & 4.69 & 1025.0 \\
200 & 11.755 & 6.50 & 4.80 & 1200.0 \\
200 & 12.02 & 6.61 & 4.91 & 1400.0 \\
200 & $\mathbf{1 2 . 2 8}$ & 6.74 & 5.03 & 1600.0 \\
200 & $\mathbf{1 2 . 5 4}$ & 6.85 & $\mathbf{5 . 1 3}$ & 1800.0 \\
200 & 12.80 & 6.96 & 5.24 & 2000.0 \\
200 & 13.02 & 7.00 & 5.34 & 2200.0 \\
200 & 13.24 & 7.10 & 5.44 & 2400.0 \\
& 13.48 & 7.20 & 5.54 & \\
\hline
\end{tabular}

normal case for all periods as evidenced by comparing case 22 with 102 , case 103 with 24 , case 25 with 104, case 105 with 27 , and case 28 with 106.

2. For a given solid low velocity layer, i.e. same thickness and velocity an increase in depth to the layer caused a decrease in deviation and an increase in the period at which a case deviated most from the normal phase velocity curve. For the liquid layer cases, an increase in depth reduced the deviation along the extremely flat portion of the phase velocity curve, but increased the deviation at longer periods, where the maximum deviation occurred. 
TABLE 2

Low Veloctty Models

All cases use the Gutenberg model, case 21, as the basic layering with a substitution of the following low velocity layers at the depths given, the remaining section being unchanged.

$d$-Layer thickness in $\mathrm{km}$.

$V_{p}$-Compression velocity in $\mathrm{km} / \mathrm{sec}$.

$V_{s}$-Shear velocity in $\mathrm{km} / \mathrm{sec}$.

$\rho$-Density in $\mathrm{g} / \mathrm{cm}^{3}$.

TMP-Total depth to mid point of low velocity layer in $\mathrm{km}$.

\begin{tabular}{|c|c|c|c|c|c|}
\hline Case & $d$ & $V_{p}$ & $V_{s}$ & $\rho$ & $T M P$ \\
\hline 101 & 40 & 6.09 & 4.00 & 3.24 & 70 \\
\hline 102 & 5 & 5.00 & 2.00 & 3.24 & 52.5 \\
\hline 22 & 10 & 5.00 & 2.00 & 3.24 & 55 \\
\hline 23 & 1 & 4.61 & 0.50 & 3.24 & 50.5 \\
\hline 14 & 1 & 4.59 & - & 3.24 & 50.5 \\
\hline 24 & 20 & 6.09 & 4.00 & 3.24 & 110 \\
\hline 103 & 40 & 6.09 & 4.00 & 3.24 & 120 \\
\hline 104 & 5 & 5.00 & 2.00 & 3.24 & 102.5 \\
\hline 25 & 10 & 5.00 & 2.00 & 3.24 & 105 \\
\hline 26 & 1 & 4.61 & 0.50 & 3.24 & 100.5 \\
\hline 15 & 1 & 4.59 & - & 3.24 & 100.5 \\
\hline 27 & 20 & 6.09 & 4.00 & 3.24 & 210 \\
\hline 105 & 40 & 6.09 & 4.00 & 3.24 & 220 \\
\hline 106 & 5 & 5.00 & 2.00 & 3.24 & 202.5 \\
\hline 28 & 10 & 5.00 & 2.00 & 3.24 & 205 \\
\hline 29 & 1 & 4.61 & 0.50 & 3.24 & 200.5 \\
\hline 16 & 1 & 4.59 & - & 3.24 & 200.5 \\
\hline
\end{tabular}

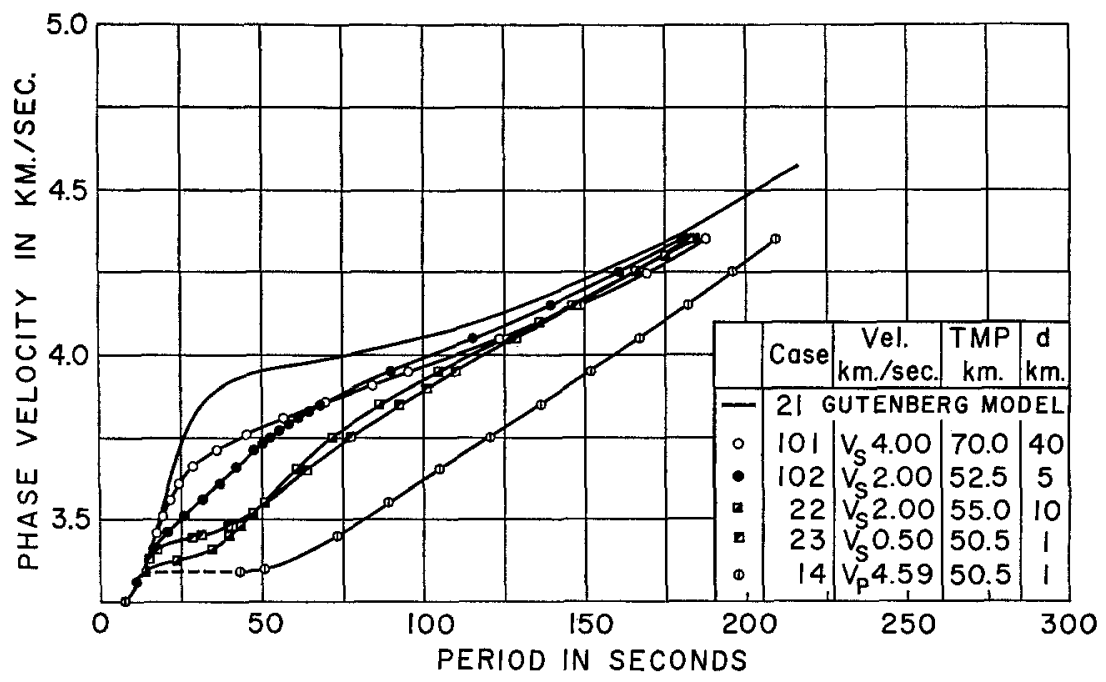

FIG. 1. Phase velocity dispersion curves of the fundamental mode for cases with an insertion of the low velocity layer at a minimum depth of 50 kilometers. 
3. Although no cases are shown, a decrease in layer velocity all other parameters being constant increases the deviation. When the velocity is decreased, (tending to increase the deviation), and the thickness is decreased (tending to decrease the

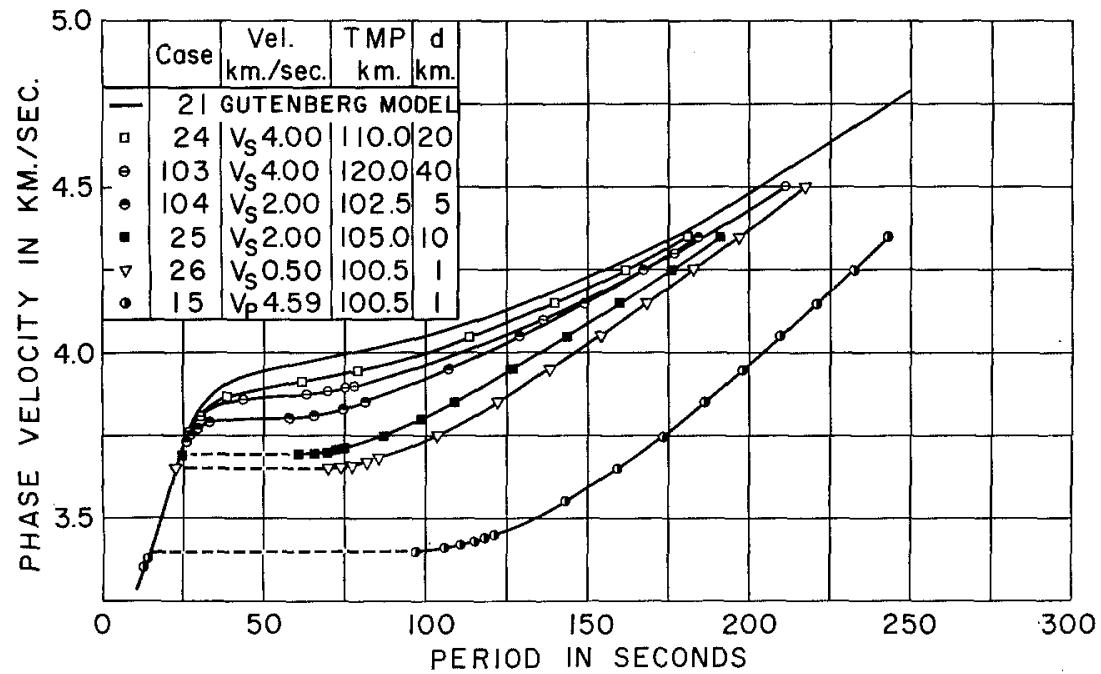

FIG. 2. Phase velocity dispersion curves of the fundamental mode for cases with an insertion of the low velocity layer at a minimum depth of 100 kilometers.

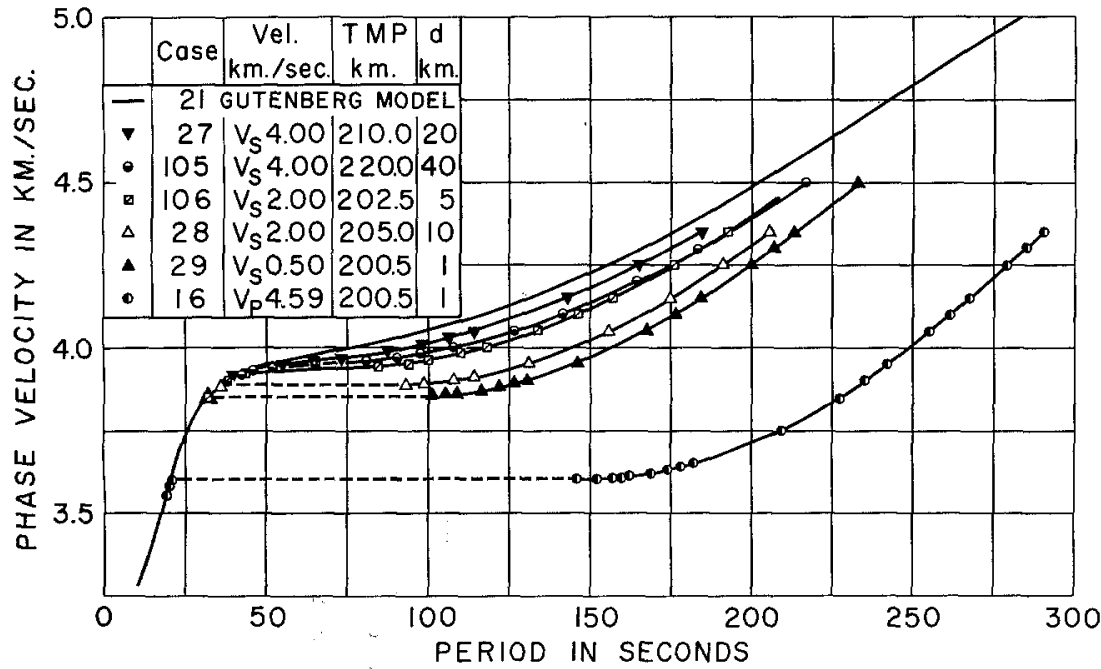

FIG. 3. Phase velocity dispersion eurves of the fundamental mode for cases with an insertion of the low velocity layer at a minimum depth of 200 kilometers.

deviation), the net effect is difficult to predict. Each situation must be studied as a special case.

4. In each of the figures, insertion of a liquid at depth produces the greatest deviation for all periods. In all cases whether liquid or solid, as the period increases past the period of maximum deviation, the phase velocities approach the normal case. 
5. The anomalous regions, described by a small variation in phase velocity with period and an associated relative maximum in group velocity, are characteristic of the soft layer models. This effect is most pronounced when the elastic velocities of the low velocity layer are smaller than the elastic velocities of the layers above it. For some of the cases the variation in phase velocity with period was so small that it was impossible to get periods in this region even with changes in phase velocity of $0.0001 \mathrm{~km} / \mathrm{sec}$. This occurred for the cases where the soft layer velocity was smaller than the layer velocities above the layer coupled with a depth to the layer of $100 \mathrm{~km}$ or greater. For these cases the phase and group velocity curves were

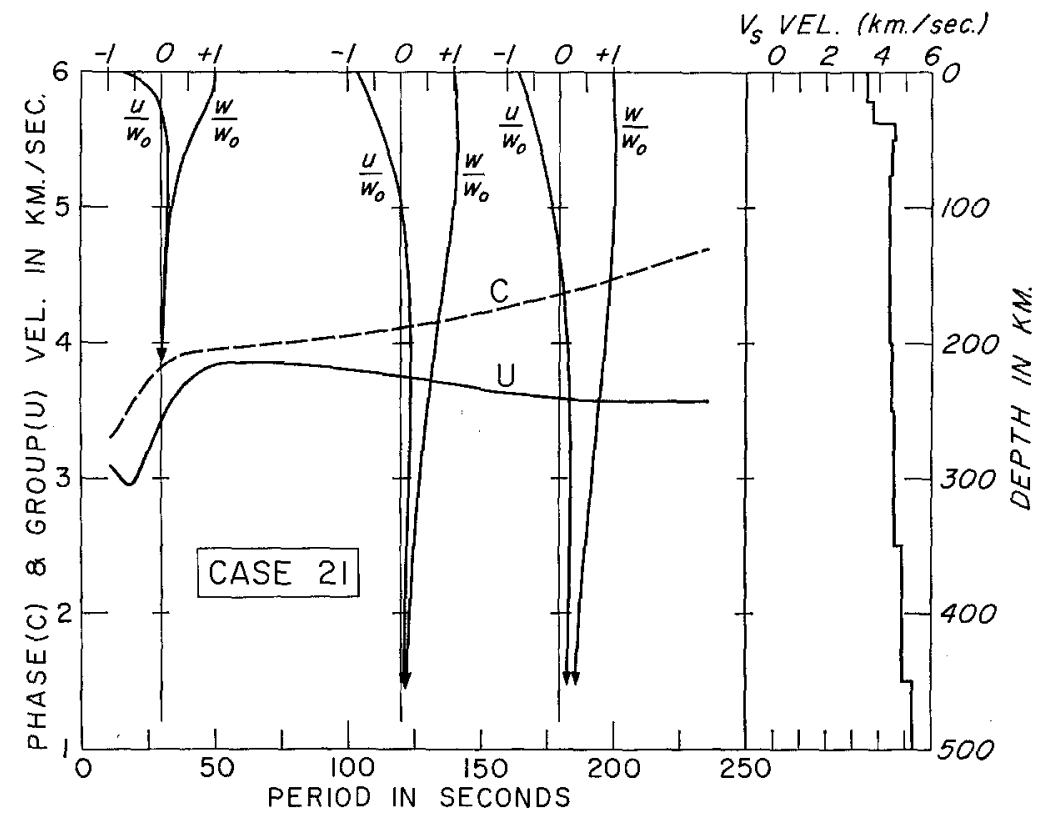

FIG. 4. Phase and group velocity dispersion curves with displacement distributions at selected periods for the fundamental mode of the Gutenberg model, case 21.

sketched in with dashed lines between the last computed points at each end of the region.

\section{Displacements at Depth}

To better understand the effects of the low velocity layer at depth on the dispersion curves, calculations of displacement versus depth were made at chosen periods for all the cases. However, because of the large number of displacement curves, we will only illustrate the results for the shallow soft layers, cases 101, 102, 22, 23, and 14 in addition to the Gutenberg model 21 . The periods were chosen so as to compare the displacements in the neighborhood of the anomalous regions of the phase velocity with the more normal parts of the dispersion curve.

The displacements versus depth curves are shown in figures $4-9$. These figures show the distribution of the vertical, $w$, and horizontal, $u$, displacements (normalized to the surface vertical displacement) superimposed on the phase and group 


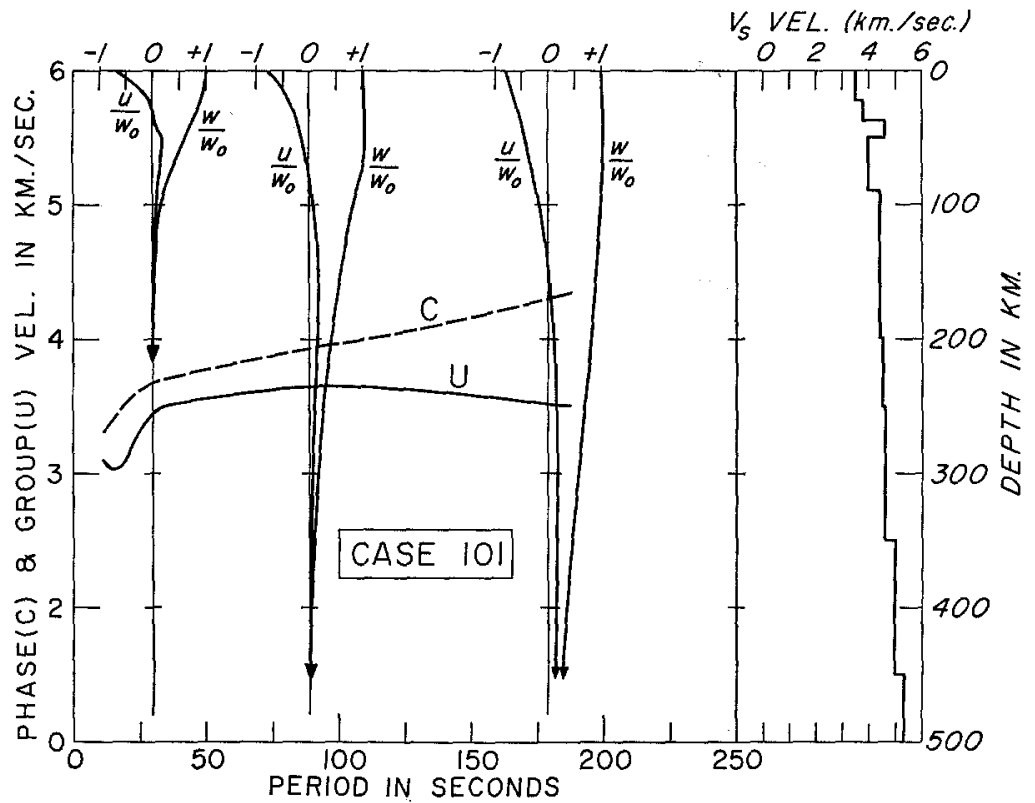

FIG. 5. Phase and group velocity dispersion curves with displacement distributions at selected periods of the fundamental mode for case 101 .

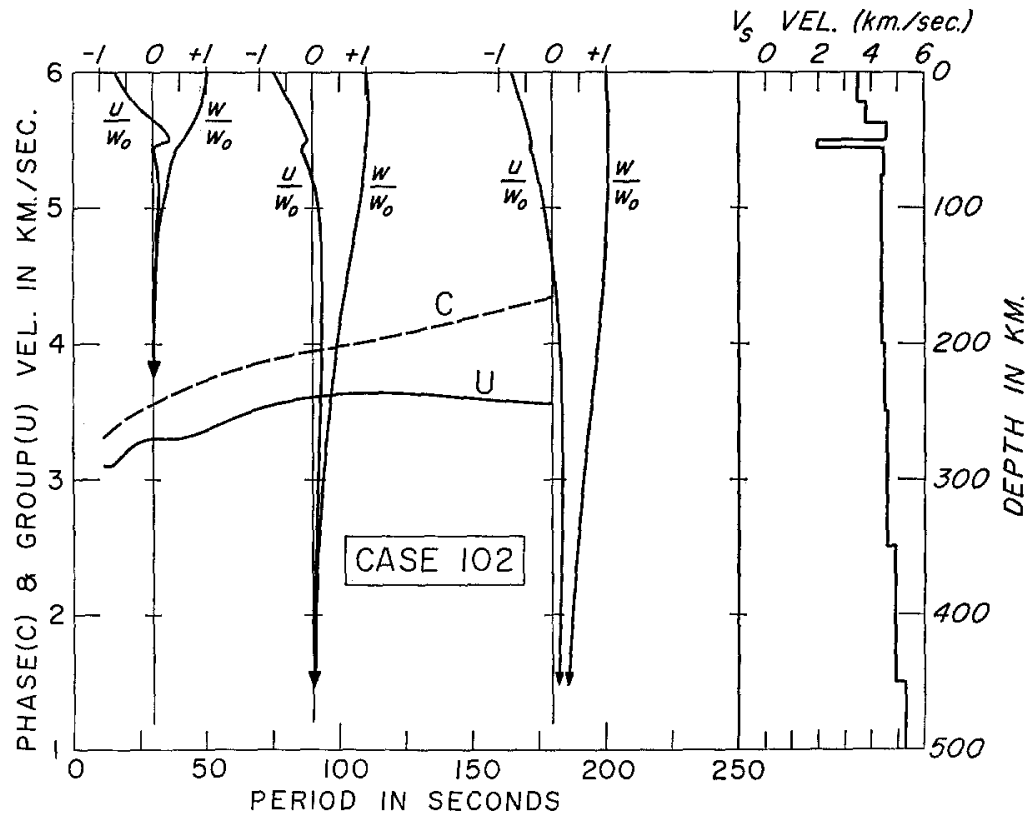

FIG. 6. Phase and group velocity dispersion curves with displacement distributions at selected periods of the fundamental mode for case 102 .

velocity curves. The displacment curves are arranged so that the zero displacement ordinate is located on the phase and group velocity curves at the period for which the displacement ratios were calculated. At the right hand side of the figure is a plot of the shear velocity versus depth. The depth scale being the same for dis- 


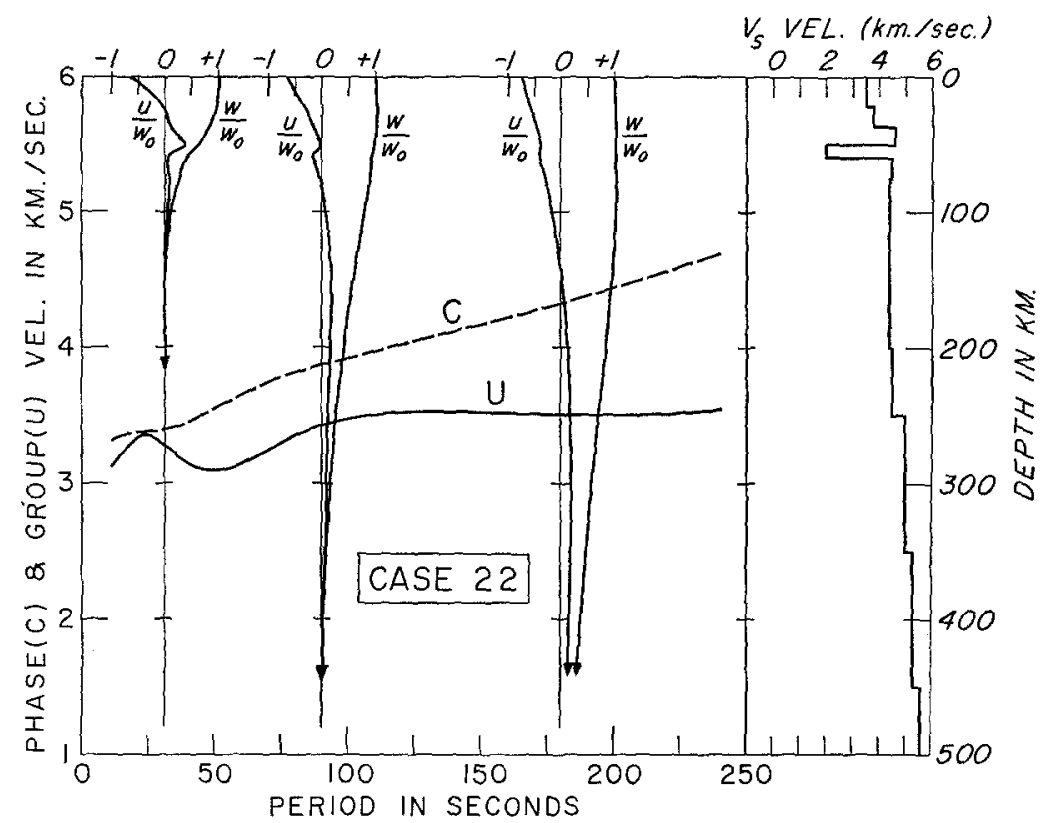

FrG. 7. Phase and group velocity dispersion curves with displacement distribution at selected periods of the fundamental mode for case 22 .

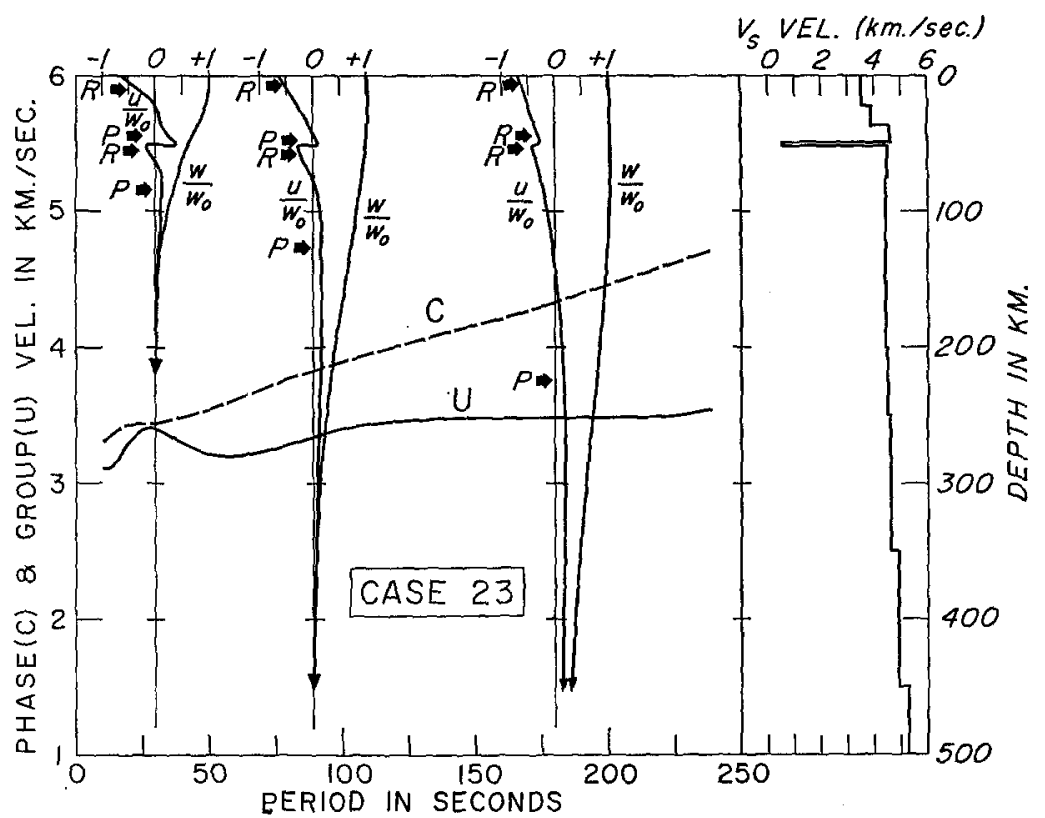

FIG. 8. Phase and group velocity dispersion curves with displacement distributions at selected periods of the fundamental mode for case 23 .

placements and velocity distribution. The following conclusions are reached from a study of these figures:

1. At periods near the anomalous parts of the dispersion curves the horizontal displacement curve has a kink associated with the low velocity zone. The vertical 
displacement is virtually unchanged. The kink in $u$ is accentuated with decreasing shear velocity or increasing thickness of the anomalous layer. In the limit of a liquid layer the kink is present for all periods and expresses the discontinuity in $u$ at a freely slipping boundary.

2. In the layers above the low velocity layer, and including part of this layer, the displacements resemble those for flexural motion in a plate as sketched in Satô (1951). For the layers below the low velocity layer the displacements have the general shape of Rayleigh waves for a velocity distribution with a free tangential stress surface located in the low velocity layer. Retrograde and prograde motion at various depths is indicated by $R$ and $P$ in figure 8 .

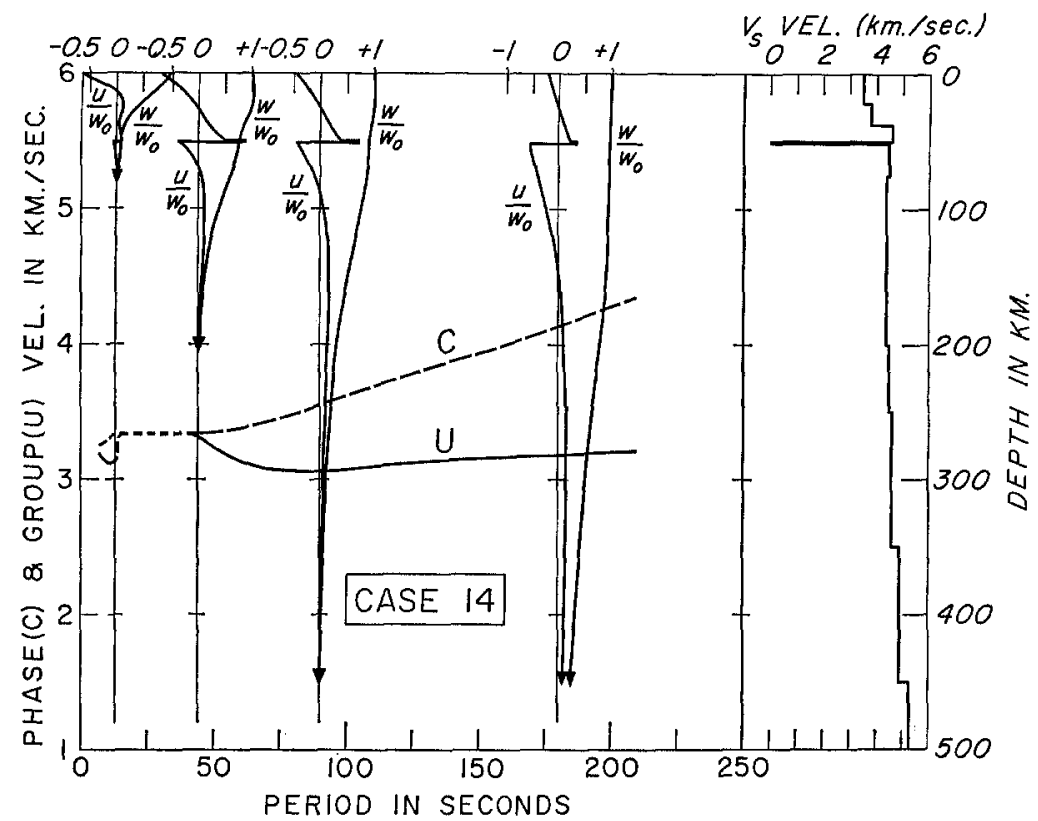

Fig. 9. Phase and group velocity dispersion curves with displacement distributions at selected periods of the fundamental mode for case 14 .

3. For periods smaller and larger than the periods for the anomalous part of the dispersion curve, the displacements for the solid low velocity layer cases are essentially the same as the displacements for the Gutenberg model, figure 4, at that period.

\section{Pseudo-Flexural Waves}

From plate theory, there are known to be real roots of the period equation for phase velocities lower than the lowest Rayleigh or body velocity in the plate. These roots correspond to flexural or antisymmetric motion in the plate. These phase velocities range from zero at an infinite period to the Rayleigh velocity in the plate at small periods. This is also true for plates over a liquid half space except, when the liquid velocity is less than the Rayleigh velocity of the plate, the short period asymptote of the real roots is the liquid-solid interface Stoneley velocity. When 
the liquid velocity is greater than the Rayleigh velocity of the plate, there are two short period real asymptotes, the Rayleigh velocity and the Stoneley velocity.

It can be shown that the period equation for a liquid at depth asymptotically factors into two period equations for wavelengths short compared to the liquid thickness. One of these period equations represents laminated or layered plates over a liquid half space and the other represents a layered half space under a liquid half space. For the first of these factors, it is then possible to have real roots corresponding to phase velocities less than the smallest Rayleigh velocity in the array. With this in mind, a search for real roots was made in the liquid at depth cases for phase velocities lower than the lowest Rayleigh velocity in the array. For all three liquid cases these roots were found and the resultant dispersion curve for case 15

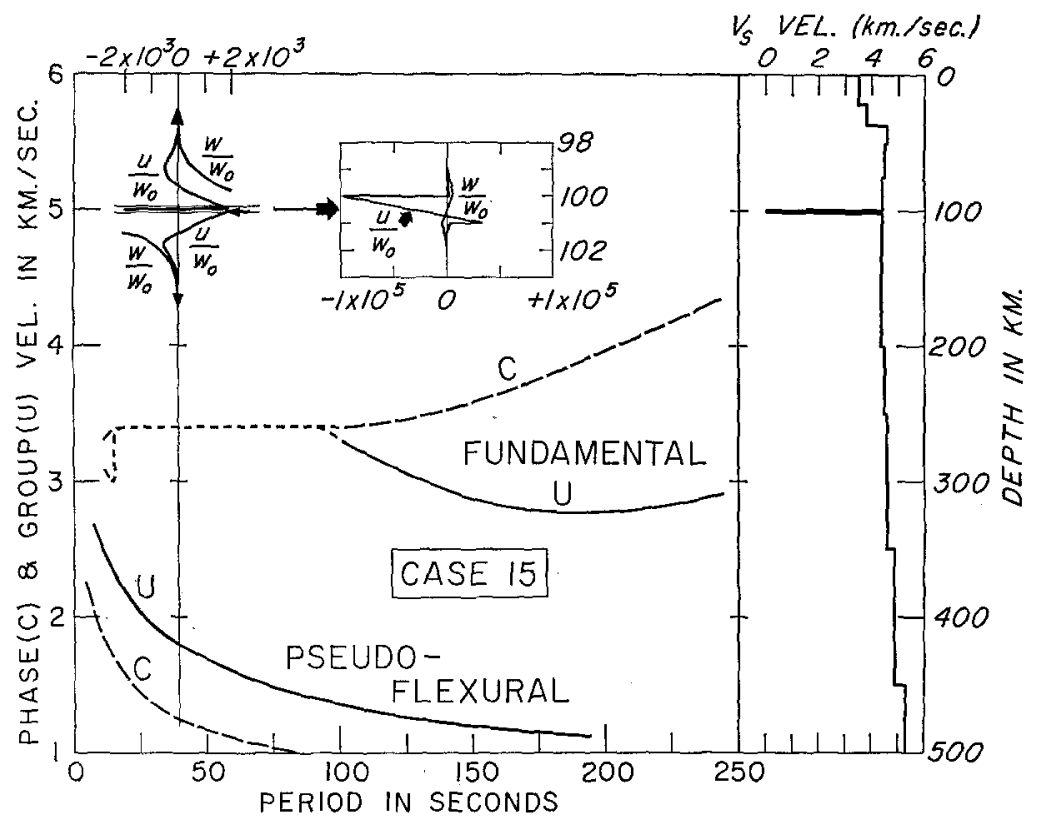

FIG. 10. Phase and group velocity dispersion eurves of the fundamental and pseudo-flexural modes with a displacement distribution of the pseudo-flexural mode for case 15 .

is shown in figure 10. A similar search was made in the solid low velocity cases, but no roots were found.

Since the displacements in the solid layers are definitely not flexural, we have chosen to call this mode the pseudo-flexural mode because the phase velocities resemble those for flexural waves in a laminated plate over a liquid half-space. The displacement for the pseudo-flexural waves decay away from the liquid layer so rapidly for all frequencies that it is highly unlikely that this mode would ever be detected at the free surface.

\section{DISCUSSION}

It is difficult to predict the elastic properties of a soft layer. We have, therefore, used a range of shear velocities $4.0,2.0,0.5$ and $0 \mathrm{~km} / \mathrm{sec}$. The highest value repre- 
sents a decrease in shear velocity of about 10 per cent from the minimal value in the normal Gutenberg model. The lowest value is consistent with a completely molten zone.

Phase velocity determinations can now be made to a precision of 1 per cent or better. With this precision every one of the models assumed can be differentiated from the normal model. The marked sensitivity of the dispersion curves to low velocities $(4 \mathrm{~km} / \mathrm{sec})$ in the mantle is evident in figures $1-3$, even when the anomalous zones are thin. The models for molten zones in the mantle, are so anomalous as to imply the possibility of detection by the phase velocity method even if their lateral extent is less than many wave lengths.

We conclude from these numerical results that the detection of soft or molten zones in the mantle by the phase velocity method is a distinct possibility. We qualify this statement to the extent that we are unable to specify the lateral extent of such zones or the shear velocity which is to be associated with them.

\section{REFERENCES}

Dorman, J., M. Ewing and J. Oliver

1960. "Study of Shear Velocity Distribution in the Upper Mantle by Mantle Rayleigh Waves", Bull.Seism.Soc.Am., 50, 87-115.

Hales, A. L.

1961. "A Weak Layer in the Mangle?" Geophys. J. R. Astr. Soc., 4, 312-319.

Harkrider, David G. and Don L. Anderson

1962. "Computation of Surface Wave Dispersion for Multilayered Anisotropic Media, Bull. Seism. Soc. Am., 52, 321-332.

Press, Frank, David Harkrider and C. A. Seafeldt

1961. "A Fast Convenient Program for Computation of Surface Wave Dispersion Curves Satô, Yasuo in Multilayered Media", Bull. Seism. Soc. Am., 51, 495-502.

1951. "Study on Surface Waves II. Velocity of Surface Waves Propagated Upon Elastic Plates", Bull. Earthq. Res. Inst., 29, 223-261.

D. G. Harkrider and F. Press

Seismological Laboratory

California Institute of Technology

Pasadena, California

A. L. Hales

Bernard Price Inst. of Geophysical Research

UNIVERSITY OF WITWATERSRAND

JoHANNESBURg, SOUTH AFrICA

Contribution 1090

Division of Geological Sciences

California Institute of Technology

Pasadena, California

Manuscript received July 13, 1962. 